\title{
Importância da utilização do teleatendimento da rede pública durante a pandemia do coronavírus: relato de experiência
}

\author{
Importance of using public telemarketing services during the coronavirus pandemic: \\ experience report
}

Importancia de utilizar los servicios públicos de telemarketing durante la pandemia de coronavirus: informe de experiência

Thais de Albuquerque Corrêa ${ }^{1 *}$, Elisama da Paz Oliveira Lima ${ }^{1}$, Renata Paula Pereira da Silva ${ }^{1}$, Aryanne Katiuska da Silva Souza ${ }^{2}$, Thais Thé Alves Carneiro ${ }^{1}$, Juliana de Alencar Ramos ${ }^{1}$, Gabriela Maria Florêncio Pereira ${ }^{1}$, Adna Karine Oliveira da Rocha ${ }^{3}$, Rayanne Victória Araújo Lins Rocha ${ }^{1}$, Tatiana Cristina Montenegro Ferreira ${ }^{1}$.

\section{RESUMO}

Objetivo: Relatar a experiência da utilização do serviço de teleatendimento da rede pública de saúde durante a pandemia do COVID-19, enfatizando sua utilidade e eficácia em tempos oportunos. Relato da experiência: A telemedicina é uma aliada importantíssima no sistema de saúde do Brasil, no enfrentamento da COVID-19, pois ela fornece serviços direcionados para a área da saúde, auxiliando o atendimento dos profissionais de saúde. Esse sistema de teleatendimento possibilitou a oferta de informações e orientações relacionadas aos sinais e sintomas de infecção por coronavírus, sem colocar em risco a vida dos envolvidos. Além disso, também proporcionou o distanciamento social no principal momento de transmissibilidade da doença. Foi utilizado o serviço por via telefônica e também através do aplicativo fornecido pela rede pública de saúde, onde, ambos se mostraram efetivos e, por meio da busca ativa, foi realizado um feedback a fim de monitorar a saúde do indivíduo que realizou o contato inicial. Considerações finais: $O$ teleatendimento se faz pauta indispensável durante a pandemia do COVID-19, tanto como solução de logística para assistência médica à distância, como, também uma possibilidade de proteção aos profissionais da saúde em faixa de risco e seus familiares, assim como aos demais da população.

Palavras-Chave: Telemedicina, Pandemias, Infecções por coronavírus.

\begin{abstract}
Objective: To report the experience of using the public health care service during the COVID-19 pandemic, emphasizing its usefulness and effectiveness in due time. Experience report: Telemedicine is a very important ally in the health system in Brazil, in coping with COVID-19, as it provides services directed to the health area, helping the care of health professionals. This call center system made it possible to offer information and guidance related to the signs and symptoms of coronavirus infection, without putting the lives of those involved at risk. In addition, it also provided social distance in the main moment of disease transmission. The service was used by telephone and also through the application provided by the public health network, where both were effective and, through the active search, feedback was carried out in order to monitor the health of the individual who made the initial contact. Final considerations: The call center is essential during the COVID-19 pandemic, both as a logistics solution for remote medical assistance, as well as a possibility of protection for health professionals at risk and their families, as well as for too much of the population.
\end{abstract}

Keywords: Telemedicine, Pandemics, Coronavirus infections.

${ }^{1}$ Faculdade Pernambucana de Saúde (FPS), Recife - PE. *E-mail: thaiscorrea_10@hotmail.com

2 Universidade de Pernambuco (UPE), Recife - PE.

3 Universidade Federal de Pernambuco (UFPE), Recife - PE. 


\section{RESUMEN}

Objetivo: Informar una experiencia de uso del servicio de salud pública durante la pandemia de COVID-19, enfatizando su utilidad y efectividad de manera oportuna. Informe de experiencia: La telemedicina es un aliado muy importante en el sistema de salud brasileño, en el enfrentamiento al COVID-19, ya que brinda servicios dirigidos al área de la salud, ayudando en la atención de los profesionales de la salud. Este sistema de call center permitió ofrecer información y orientación relacionada con los signos y síntomas de la infección por coronavirus, sin poner en riesgo la vida de los involucrados. Además, también brindó distancia social en el momento principal de transmisión de la enfermedad. El servicio se utilizó por teléfono y también a través de una aplicación de la red de salud pública, donde ambos resultaron efectivos y, mediante búsqueda activa, se realizó para monitorear la salud del individuo que hizo el contacto inicial. Consideraciones finales: El call center es fundamental durante una pandemia de COVID-19, tanto como solución logística para la atención médica remota, como la posibilidad de protección de la salud profesional en riesgo y sus familias, así como para gran parte de la población.

Palabras clave: Telemedicina, Pandemias, Infecciones por coronavirus.

\section{INTRODUÇÃO}

Os coronavírus (com sua morfologia esférica e projeções de face parecido a uma coroa) são grandes vírus de RNA de fita simples, positivos, que infectam uma grande diversidade de animais e também humanos. $O$ SARS-CoV-2 (COVID-19) aparentemente teve sucesso em fazer sua transferência de animais para humanos no mercado de frutos do mar de Huanan em Wuhan, China, e foi aí onde tudo começou (VELAVAN TP E MEYER CG, 2020).

A pandemia do novo Corona vírus ocasionou e está ocasionando um grande impacto em todos os pilares da sociedade, levando a população a experienciar momentos de incertezas, pânico e medo, principalmente devido à inexistência de um tratamento comprovadamente eficaz e seguro (PORTNOY J, et al., 2020).

A dificuldade que os sistemas de saúde sofrem é uma adversidade muito complexa e difícil visto que os serviços são fornecidos não só para os indivíduos que são infectados pelo COVID-19, mas também para todas as outras pessoas que sofrem de patologias mais graves, tanto elas agudas como crônicas, e, além disso, pacientes traumáticos. Nesse cenário, torna-se delicada e minuciosa a proteção e preservação dos médicos, enfermeiras e outros profissionais da área da saúde. Com isso, os sistemas de saúde do mundo inteiro estão empregando em seus serviços a telemedicina com a finalidade de proporcionar cuidados, porém, mantendo os usuários e pacientes em suas casas, preservando assim a saúde e integridade de todos. Esta transformação acentuada para a telemedicina certifica sua proficiência e valor, trazendo-a como um instrumento competente para o denominado distanciamento social em espaços clínicos ou outros (BASHSHUR R, et al., 2020).

Podemos definir telemedicina como a utilização de tecnologias de informação e comunicação na saúde (principalmente em situações onde a distância é um elemento determinante) onde é possível proporcionar a entrega de serviços voltados aos cuidados na área da saúde (MALDONADO JMSDV, et al., 2016). Ela aparece como um guia na grande batalha a ser vencida provocada pelo novo coronavírus, além de ter transformado e remodelado a relação médico-paciente (BRITO MEDEIROS AM, et al., 2020). Esse sistema tem o potencial de ajudar pacientes levemente enfermos a obter os cuidados de suporte que precisam, minimizando sua exposição a outros pacientes com doenças agudas (PORTNOY J, et al., 2020).

Uma pesquisa recente demonstrou que os pacientes estão dispostos a usar a telessaúde, mas ainda existem barreiras, a saber: em um momento de necessidade, muitas pessoas voltam ao que estão acostumadas a fazer e à maneira como interagiam anteriormente com o sistema de saúde; os pacientes preferem ser atendidos de forma presencial por se sentirem mais seguros visto que serão examinados e, além de tudo, há indivíduos que não sabem como acessar o sistema (PORTNOY J, et al., 2020).

A telemedicina é uma aliada importantíssima no sistema de saúde do Brasil, principalmente no enfrentamento do COVID-19, pois ela fornece serviços direcionados para o setor da saúde, auxiliando o 
atendimento dos profissionais. Além disso, durante esse período pontual, esse sistema foi indispensável na comunicação dos usuários para com os profissionais sem precisar realizar o deslocamento até o hospital, conseguindo levar mais conforto e paz para o equilíbrio emocional dos indivíduos (BRITO BO e LEITÃO LPC, 2020).

Desta forma, o presente estudo teve como objetivo relatar a experiência da utilização do serviço de teleatendimento da rede pública de saúde durante a pandemia do COVID-19, enfatizando sua utilidade e eficácia em tempos oportunos.

\section{RELATO DE EXPERIÊNCIA}

Foi desenvolvido um relato de experiência vivenciado por uma estudante de enfermagem na utilização do teleatendimento da rede pública de saúde durante o período de quarentena da pandemia do COVID-19, no estado de Pernambuco, em abril de 2020. Foi perceptível que o atendimento proporcionou o distanciamento social no principal momento de transmissibilidade da doença.

Os serviços utilizados foram: via telefônica e aplicativo fornecido pela rede pública de saúde. Ambos se mostraram efetivos e, por meio da busca ativa, foi realizado um feedback (o que estreitou mais ainda a relação paciente-profissional) a fim de monitorar a saúde daquele indivíduo que realizou o contato inicial.

O feedback foi indispensável nesse processo de comunicação paciente-profissional, pois, com isso, foi possível orientar, avaliar, acompanhar e acalmar o enfermo durante seus sentimentos apreensivos relacionados ao vírus e aos seus sintomas pessoais. Além do mais, o feedback consegue deixar o indivíduo mais sossegado e despreocupado, afastando a ansiedade que poderia piorar o seu estado de saúde.

Foi notório a melhora do bem-estar e do ânimo do usuário no momento de utilização do serviço. Durante o contato, foram feitas várias perguntas a fim de saber o estado do paciente de uma forma detalhada, onde, as principais foram: "quais são seus sintomas e quando começaram? qual sua idade? mora sozinha (o)? qual o hospital mais próximo de sua residência? tem doenças crônicas? está praticando o isolamento social? trabalha? ".

$O$ atendimento por via telefônica transmitiu mais tranquilidade, pois, ambos (paciente e profissional) puderam conversar de forma mais aberta e estreita. Além disso, a escuta do tom de voz do paciente ajuda o profissional a perceber quão grave é o estado de saúde do mesmo naquele momento. Já o aplicativo, serviu como uma forma de monitoramento e rastreamento.

Após vários questionamentos por parte do profissional de saúde, foram dadas várias orientações de como o enfermo deveria prosseguir, e, algumas delas foram: procurar atendimento médico se houver falta de ar ou febre, aumentar a ingesta hídrica, escolher alimentos saudáveis e que fortalecessem o sistema imunológico, observar a progressão ou regressão dos sinais e sintomas, realizar o distanciamento social (se possível) e tomar as medidas preventivas adequadas (lavar frequentemente as mãos, usar a máscara facial e trocá-la a cada 2 horas e separar as roupas contaminadas em um só ambiente).

O uso do teleatendimento conseguiu suprir as necessidades de informação do indivíduo que não podia se locomover devido às superlotações hospitalares do início da pandemia. Além disso, muitas pessoas que utilizaram o serviço não sabiam ao certo se realmente estavam com COVID-19, e, se realmente fossem aos pontos de atendimento presenciais, poderiam ser colocadas em um risco maior. Os casos mais graves não conseguiam ser solucionados de forma remota, sendo necessário se dirigir diretamente aos hospitais.

Em contrapartida, o fato de saber que o profissional de saúde não estaria fazendo uma avaliação realmente adequada (sem observar os sinais vitais e sem fazer o exame físico) evidenciou preocupação ao longo da utilização do serviço, podendo ser considerado como um ponto negativo, pois o exame físico é algo imprescindível durante um atendimento, visto que observa como o paciente se encontra, tendo uma visão mais detalhada de seus sintomas. É importante verificar como está o funcionamento dos sistemas do paciente (principalmente o sistema cardiovascular e respiratório, que são os mais afetados pelo vírus do COVID-19) realizando ausculta, palpação, percussão e inspeção. Porém, isso não é possível através do teleatendimento, e, devido a isso, o paciente fica mais vulnerável. 


\section{DISCUSSÃO}

Com o início da pandemia em 11 de março de 2020, quando a Organização mundial de Saúde declarou surto da doença COVID-19, a estratégia de resposta incluiu diagnóstico precoce, isolamento do paciente, monitoramento sintomático de casos de contato, bem como casos suspeitos e confirmados, e quarentena, tudo isso com o intuito de diminuir a transmissibilidade da doença e tentar, de alguma forma, controlar o vírus (OHANNESSIAN R, et al., 2020).

Os sintomas associados ao novo coronavírus podem ser diversificados, podendo ser um simples resfriado ou agravar para uma pneumonia severa. O paciente pode apresentar febre, tosse, dificuldade de respirar, dor muscular, confusão mental, dor de cabeça, dor de garganta, rinorreia, dor no peito, diarreia, náusea e vômito. Além disso, o sistema respiratório tornou-se o mais referido entre os sinais e sintomas associados ao COVID19, levando o paciente a procurar o serviço de telemedicina a partir do surgimento das primeiras manifestações clínicas (BAÊTA KF, et al., 2020). Neste contexto, a telemedicina tem sido promovida e ampliada para reduzir o risco de transmissão, sendo uma solução telemédica para superar limitações pessoais, técnicas e de infraestrutura.

Esse sistema de teleatendimento mostrou-se eficaz em surtos anteriores, incluindo surtos antigos de coronavírus, como SARS-CoV (coronavírus associado à síndrome respiratória aguda grave) e MERS-CoV (síndrome respiratória do Oriente Médio coronavírus). A telemedicina consegue realizar o monitoramento de forma remota de pacientes de contato e teleconsulta para triagem, pois, com o aumento da tecnologia e disseminação em massa de smartphones foi possível realizar sua implementação mais rapidamente. Por outro lado, é perceptível que a maior parte do público da terceira idade (idosos) tem uma certa dificuldade no manuseio da tecnologia, e, infelizmente, é um dos grupos mais acometidos pela doença (LINS AF, et al., 2019; OHANNESSIAN R, et al., 2020).

A telemedicina possibilitou a oferta de informações e orientações relacionadas aos sinais e sintomas de infecção por corona vírus sem colocar em risco a vida dos envolvidos, assegurando uma certa tranquilidade por fornecer dicas, aconselhamentos e acolhimento ao indivíduo, restabelecendo um pouco da saúde mental do usuário que se encontrava em extremo esgotamento emocional. Em contrapartida, é reconhecido que esse sistema não proporciona o exame físico do paciente por ser um serviço à distância, o que compromete um pouco a assistência correta, tal achado corroborando com outros estudos. Porém, é fato que o exame físico é algo que pode ter um efeito substancial no atendimento, pois, é importante nas interações médico-paciente, além de ser um auxiliar relevante para o diagnóstico clínico preciso e pode ser ensinado de forma eficaz usando dicas práticas (MARTíNEZ-GARCÍA, et al., 2020; MEDEIROS LGD, et al., 2020; STEINMAN et al., 2015).

Mesmo com o progresso tecnológico possibilitando inúmeros avanços relevantes na concretização de diagnósticos mais objetivos, ainda assim, a entrevista clínica segue como um bom mecanismo de localizar os principais problemas do paciente, pois, o cliente é, sem dúvidas, especialista de seus próprios sentimentos e sintomas, pertencendo ao professional de saúde a responsabilidade de entender, escutar e sentir toda a história a respeito da doença. Com isso, a comunicação inteligente e eficiente em uma consulta se torna um dos pontos chaves para a revelação e manifestação de certas comorbidades. Neste quesito, a telemedicina deixa a desejar por não ser capaz de realizar a entrevista clínica e realizar o exame físico do paciente, como dito anteriormente (SANTOS MCD, et al., 2010).

Foi notório que o atendimento online proporcionou o distanciamento social no principal momento de transmissibilidade da doença, permitindo, assim, uma consulta virtual, não presencial e satisfatória. Com isso, foi dado uma maior atenção aos problemas agudos, acompanhamento dos pacientes à distância, promoção e prevenção à saúde. Isso é possível quando se tem profissionais capacitados a realizarem uma consulta à distância de qualidade, pois, quando preparados, é perceptível o manejo correto à determinadas situações, além de possuírem conhecimentos sólidos com discernimento, prudência, compaixão e interesse no cuidado dos pacientes. Ainda assim, é sempre válido a realização de treinamento para os profissionais que fazem uso da telemedicina, objetivando diminuir os problemas de falta de qualidade dos dados fornecidos e recebidos (OLIVEIRA AB, et al., 2020). 
Os sistemas de atendimento remoto podem melhorar significativamente a triagem, tratamento e coordenação de cuidados para pacientes com COVID-19 confirmado, especialmente em áreas de poucos recursos. Mesmo os prestadores de cuidados de saúde que ainda não adotaram recursos de telemedicina devem considerar a implementação de forma restrita com sistemas projetados para mitigar o aumento esperado nos volumes de pacientes de baixa acuidade que buscam segurança (ROCKWELL KL, GILROY AS, 2020).

O teleatendimento se fez e faz pauta indispensável durante a pandemia do COVID-19, tanto como solução de logística para assistência médica à distância, como uma possibilidade de proteção aos profissionais da saúde em faixa de risco e seus familiares, assim como aos demais da população, além de minimizar o nervosismo e a ansiedade ao longo da pandemia em si. Os casos leves de COVID-19 podem ter telemonitoramento que alerta em tempo hábil a necessidade de internação, sem exposição a potencial contágio. Além disso, as consultas por videochamada também podem ajudar muito no acompanhamento de paciente com doenças crônicas, como diabetes e hipertensão (QUISPE-JULI C, et al., 2020).

Desta maneira é nítido que à medida que a pandemia avança, o interesse, a busca e o uso de ferramentas digitais voltados para a área da saúde aumentam, visto que os registros eletrônicos médicos conseguem permitir o gerenciamento oportuno de informações para auxiliar a tomada de decisão. A telemedicina possibilita aos usuários não só mais conforto, como também segurança e praticidade, acarretando também mais comodidade e prevenção para os priofissionais de saúde durante todo o curso da pandemia do COVID19. Portanto, é nítida a importância do incentivo e da prática ao uso da telemedicina em tempos oportunos. Como limitações do estudo foi nítido a ansiedade devido à preocupação de ter adquirido o vírus.

\section{REFERÊNCIAS}

1. BAÊTA KF, et al. Protocolo de Tratamento do Novo Coronavírus (2019-nCoV), 2020.

2. BASHSHUR R, et al. Telemedicine and the COVID-19 pandemic, lessons for the future. Guest Editorial, 2020; 6(5).

3. BRITO MEDEIROS AM, et al. Os desafios da telemedicina frente a relação médico-paciente em tempos de Covid19. Research, Society and Development, 2020; 9(10):9219109350-9219109350.

4. LINS AF, et al. O uso da telemedicina como ferramenta para aprimorar os serviços de saúde: viabilidade e desafios. Revista Educação em Saúde, 2019; 7(1).

5. MALDONADO JMSDV, et al. Telemedicina: desafios à sua difusão no Brasil. Cadernos de Saúde Pública, 2016; 32: e00155615.

6. MARTÍNEZ-GARCÍA M, et al. Telemedicina con telemonitorización en el seguimiento de pacientes con COVID-19. Revista Clínica Espanola, 2020; 220(8): 472-479.

7. MEDEIROS LGD, et al. Telemedicina - O "NOVO NORMAL" do atendimento aos pacientes e colaboradores de centro oncohematológico, em tempos de COVID-19: Relato de Experiência. Hematology, Transfusion and Cell Therapy, 2020; $42: 559$.

8. OHANNESSIAN R, et al. Global telemedicine implementation and integration within health systems to fight the COVID19 pandemic: a call to action. JMIR public health and surveillance, 2020; 6(2): 18810.

9. OLIVEIRA AB, et al. Desafios do avanço da Telemedicina e seus aspectos éticos: revisão integrativa. Comunicação em ciências da saúde, 2020; 31(1): 55-63.

10. BRITO BO, LEITÃO LPC. Telemedicina como estratégia de combate a COVID-19 na região norte do brasil. Saúde em redes, 2020; 6(2).

11. PEREIRA MC, et al. Telessaúde e Covid-19: experiências da enfermagem e psicologia. Revista de Saúde Pública do Paraná, 2020.

12. PORTNOY J, et al. Telemedicine in the era of COVID-19. The Journal of Allergy and Clinical Immunology: In Practice, 2020; 8(5): 1489-1491.

13. QUISPE-JULI C, et al. COVID-19: Una pandemia en la era de la salud digital, 2020.

14. ROCKWELL KL, GILROY AS. Incorporating telemedicine as part of COVID-19 outbreak response systems. American Journal of Managed Care, 2020; 26(4): 147-148.

15. SANTOS MCD, et al. Comunicação em saúde e a segurança do doente: problemas e desafios. Revista portuguesa de saúde pública, 2010; 47:57.

16. STEINMAN M, et al. Impacto da telemedicina na cultura hospitalar e suas consequências na qualidade e segurança do cuidado. Einstein (São Paulo), 2015; 13 (4): 580-586.

17. VELAVAN TP, MEYER CG. The COVID-19 epidemic. Tropical medicine \& international health, 2020; 25(3): 278. 